\title{
Metformin Combined with Alendronate Ameliorates Osteoarthritis by Attenuating RANKL-induced Bone Resorption and Protecting Cartilage Against Degradation
}

\section{Yanjing Guo}

Shanxi Medical University

\section{Ruijia Yang}

Shanxi Medical University

\section{Sujing Zong}

Shanxi Medical University

\section{Zhenyu Wang}

The Second Hospital of Shanxi Medical University

Jiyu Zhao

The Second Hospital of Shanxi Medical University

\section{Chongwei Chen}

The Second Hospital of Shanxi Medical University

\section{Chunfang Wang}

The Second Hospital of Shanxi Medical University

Shaowei Wang ( $\nabla$ wangshaowei2010@126.com)

The Second Hospital of Shanxi Medical University

\section{Research Article}

Keywords: osteoarthritis, subchondral bone, cartilage, metformin, alendronate

Posted Date: July 28th, 2022

DOl: https://doi.org/10.21203/rs.3.rs-1378528/v2

License: (c) (i) This work is licensed under a Creative Commons Attribution 4.0 International License. Read Full License 


\section{Abstract}

The purpose of the present study was to investigate the effect of metformin combined with alendronate on subchondral bone and cartilage degeneration in a destabilization of the medial meniscus (DMM)induced osteoarthritis (OA) mouse model. There were lower OARSI scores and fewer TRAP-positive cells in DMM mice after treatment with metformin combined with alendronate. In cartilage, metformin inhibited the expression of MMP3 and MMP13 in IL-1 $\beta$-stimulated OA chondrocytes and increased the expression of Sox9 and aggrecan. In parallel, metformin also inhibited cartilage matrix degradation, as shown by Alcian blue staining and SO/FG staining in ATDC5 cells and cartilage tissues. Moreover, metformin and alendronate downregulated the levels of specific genes and proteins (NFATc1, c-Fos, DCSTAMP, TNFRSF11, CTSK), thereby inhibiting osteoclast differentiation in vitro. Mechanistically, metformin increased the expression of phospho-AMPK alpha1, attenuated the phosphorylation of NF-KB, and reduced reactive oxygen species (ROS) levels. Metformin combined with alendronate may be a useful candidate for attenuating early-stage $O A$. This would be an ideal drug/drug combination for treating osteoarthritis in the presence of osteoporosis combined with T2D.

\section{Introduction}

Osteoarthritis $(\mathrm{OA})$ is a chronic joint disease characterized by cartilage degeneration and subchondral bone remodeling [1]. The development of osteoarthritis is associated with several factors, including aging, joint inflammation, and joint instability, and is driven by biological pathways within the joint and biomechanical factors[2]. One recent meta-analysis suggested that patients with type 2 diabetes (T2D) were more likely to develop knee OA than non-diabetic patients[3]. Moreover, the biomechanical quality of bone is compromised in diabetic patients, so T2D often cooccurs with osteoporosis[4]. Alendronate, which inhibits bone resorption, is therefore the main choice for treating osteoporosis. In a large retrospective case-control study, the incidence of T2D was reduced in patients with osteoporosis who were treated with alendronate[5]. Metformin is the first-line therapy for diabetes mellitus type 2 when obesity is present. Due to its protective effect on the skeletal system, metformin is used preferentially for treating T2D in patients with osteoporosis.

In a cohort study, patients with OA and T2DM were treated with a combination of COX-2 inhibitors and metformin, and these patients had a lower rate of joint replacement surgery than those who were not treated with the combination[6]. This outcome may be because the combination treatment reduced the associated proinflammatory factors more than in patients who were not treated with metformin. Therefore, metformin may be a potential candidate for the development of effective treatments for OA. The antidiabetic effect of metformin is mainly achieved by activating AMP-activated protein kinase (AMPK) through the stimulation of Thr-172 phosphorylation on AMP-activated protein kinase alpha1 (AMPKa1)[7]. AMPK is a chondrocyte bioenergy sensor that has been suggested to be involved in OA development[8], and its activity is decreased in human knee OA chondrocytes and cartilage[9]. Knockout of AMPKa1, the catalytic alpha subunit of AMPK, in mice accelerates the progression of osteoarthritis induced by destabilization of the medial meniscus surgery[10]. These findings indicated that maintaining 
AMPK activity in chondrocytes may be important in delaying cartilage damage. Recently, metformin has been proven to have anti-inflammatory effects in treating OA. Some studies have shown that metformin enhances AMPK phosphorylation in chondrocytes and subsequently delays OA development in a mouse destabilization of the medial meniscus (DMM) model[11]. When AMPKa1 was knocked out, the chondroprotective effect of metformin was lost[11]. This finding means that a chondrocyte-targeted version of metformin can be used to treat OA. Furthermore, it has been shown that AMPK negatively regulates the differentiation and function of osteoclasts[12].

The subchondral bone plays a significant role in joint homeostasis and OA development[13]. The structural alterations in subchondral bone in $\mathrm{OA}$ are thought to enhance crosstalk with articular cartilage and therefore accelerate the progression of OA[14]. Animal studies have shown that changes in subchondral bone, such as increased osteoclast activity and bone remodeling, might occur at the same time as changes in cartilage[15]. In a study in which meniscectomized mice were treated with OPG, an inhibitor of osteoclastic bone resorption activity, cartilage destruction in the mice was abrogated[16]. Alendronate, a bisphosphonate, also limits the progression of $\mathrm{OA}$ by enhancing subchondral bone mass by inhibiting osteoclasts and bone resorption[17]. These studies suggest that restoring abnormal subchondral bone reconstruction by inhibiting osteoclastic bone resorption in the early stages of $O A$ could be a therapeutic strategy for OA. However, there is a risk that long-term use of alendronate may alter bone strength due to excessive inhibition of bone transformation[18].

In the present study, we investigated the therapeutic effect of low concentrations of alendronate combined with metformin and administered at low frequency in a murine model of OA induced by surgical destabilization of the medial meniscus. We found that low concentrations of alendronate counteracted DMM-induced subchondral bone loss and cartilage degeneration and that these effects were enhanced when alendronate was combined with metformin. Furthermore, the mechanism by which metformin affected the AMPK signaling pathway was analyzed. In addition, metformin combined with alendronate may delay the progression of osteoarthritis in patients with T2D combined with osteoporosis.

\section{Method}

\section{Mice}

OA was induced in 8-week-old male C57BL/6 mice by destabilization of the medial meniscus (DMM) in the right knee, followed by random allocation into a control group and two treatment groups: treatment with alendronate alone or alendronate plus metformin ( $n=5$ per group). The day after surgery, Group A received 2 intraperitoneal injections of alendronate (APExBIO, CAS No. 121268-17-5) weekly for 8 weeks at a dose of $0.1 \mathrm{mg} / \mathrm{kg}$. Group B received alendronate plus $205 \mathrm{mg} / \mathrm{kg}$ metformin (CSNpharm, CAS No. 627-24-9) in the same manner.

\section{Cell culture}


We purchased the murine ATDC5 cell line from Shanghai Jining Industrial Co. The cells were cultured in a complete medium consisting of DMEM/F12 (HyClone), 1\% penicillin/streptomycin (Sigma), and 10\% fetal bovine serum (CellMax). We changed the medium every 2 days. The cells were passaged when the cells were $80 \%-90 \%$ confluent.

Bone marrow monocytes (BMMs) were isolated from the tibia and femur marrow of C57BL/ 6 mice. BMMs were maintained in complete medium consisting of alpha-modified minimal essential medium (aMEM) (HyClone) containing 10\% fetal bovine serum (vwr), 1\% penicillin/streptomycin (Sigma) and 25 $\mathrm{ng} / \mathrm{mL}$ macrophage-colony-stimulating factor (M-CSF) (LifeTein, New Jersey, USA). Afterward, the BMMs were stimulated with $50 \mathrm{ng} / \mathrm{mL}$ receptor activator of nuclear factor-kB ligand (RANKL) (LifeTein, New Jersey, USA) and $25 \mathrm{ng} / \mathrm{mL}$ M-CSF.

\section{Cell treatment and CCK-8 assay}

We used different concentrations of metformin $(0,2,4$, and $5 \mathrm{mmol} / \mathrm{L})$ to treat the murine ATDC5 cell line with or without $10 \mathrm{ng} / \mathrm{mL}$ recombinant murine interleukin (IL)-1 (PeproTech, Rocky Hill, NJ, USA).

BMMs were cultured with varying concentrations of alendronate $(0,62.5,125 \mathrm{nmol} / \mathrm{L})$ or metformin $(0$, $62.5,125 \mu \mathrm{mol} / \mathrm{L}$ ) in the presence of $100 \mathrm{ng} / \mathrm{mL}$ RANKL.

For CCK-8 assays, the cells were seeded into 96-well plates. After adherence, the cells were treated with different concentrations of metformin or alendronate. Two days later, $10 \mu \mathrm{l}$ of CCK-8 solution (JoyTech Bio, Zhejiang, China) was added to the wells and incubated for $2 \mathrm{~h}$. The optical density at $450 \mathrm{~nm}$ was measured using a microplate reader.

\section{High-density culture of the murine ATDC5 cell line}

EDTA-trypsin (Gibco) was used to digest the cells, and the cells were resuspended at a concentration of 2 $x 10^{6} / \mathrm{mL}$. Then, $10 \mu \mathrm{l}$ of the cell suspension was added dropwise at hourly intervals to a 24 -well plate, 4 times in total. Then, $500 \mu \mathrm{L}$ of complete medium was added to each well with or without $10 \mathrm{ng} / \mathrm{mL} \mathrm{IL}-$ $1 \beta$ and incubated for 6 days. Moreover, different concentrations of metformin $(0,2,4$, and $5 \mathrm{mmol} / \mathrm{L})$ were added to the cultured cells. The medium was changed every other day. The chondrogenic matrix was examined by Alcian blue staining. The stained area was scanned by the scanner and measured with ImageJ.

\section{Organoid culture}

The "relatively normal" cartilage tissue from the tibial plateau of a total knee replacement patient was cut into small pieces and cultured in DMEM/F12 supplemented with $10 \%$ FBS with various concentrations of metformin $(0,2,4$, and $5 \mathrm{mmol} / \mathrm{L}$ with or without IL-1 $\beta(10 \mathrm{ng} / \mathrm{mL}))$. The cartilage pieces were collected at 24,48 , and $72 \mathrm{~h}$. After $16 \mathrm{~h}$ of fixation with $4 \%$ paraformaldehyde, the sample was dehydrated through a graded series of ethanol, cleared, and embedded in paraffin. Then, 5- $\mu \mathrm{m}$-thick sections were cut and collected on glass slides. Integrated optical density 
values of proteoglycan content were determined by Safranin O-fast green and immunohistochemical staining.

\section{The efficacy of metformin and alendronate on osteoclasts}

BMMs were seeded at a density of $8 \times 10^{3}$ cells/well in a 96-well plate with complete alphaMEM containing RANKL $(50 \mathrm{ng} / \mathrm{mL})$ in the presence of metformin $(0,62.5$, and $125 \mu \mathrm{mol} / \mathrm{L})$ or alendronate $(0,62.5$ and $125 \mathrm{nmol} / \mathrm{L})$ for 7 days. The medium was changed every 2 days.

After osteoclasts formed, the differentiated osteoclasts were fixed with $4 \%$ paraformaldehyde and stained for tartrate-resistant acid phosphatase (TRAP) (JoyTech Bio, Zhejiang, China). We evaluated the differentiation of osteoclasts by counting TRAP-positive multinucleated cells (MNCs) that contained 3 or more nuclei.

\section{Quantitative Real-Time Polymerase Chain Reaction (qRT-PCR)}

After 7 days of stimulation with metformin $(0,2,4,5 \mathrm{mmol} / \mathrm{L})$ in the presence or absence of IL-1 3 , we extracted total RNA from chondrocytes with TRIzol reagent (Takara, Japan). The RNA concentration was determined using NanoDrop one at $260 \mathrm{~nm}$. Then $1 \mu \mathrm{g}$ of total RNA was reverse transcribed to complementary DNA (cDNA) using a reverse transcription kit (Takara, Japan). The expression of target genes was examined using a QuantiTect SYBR Green PCR kit (Takara, Japan) with an ABI QuantStudio 6 Real-Time PCR System (Thermo Fisher Scientific, Waltham, MA, USA). Changes in the target genes were analyzed by the $2-\Delta \Delta \mathrm{Cq}$ method. We used the expression of the housekeeping gene 18S RNA as the reference for our analysis.

A total of $1.5 \times 10^{5}$ primary BMMs per well were inoculated in a 6-well plate and stimulated with RANKL $(50 \mathrm{ng} / \mathrm{mL})$ and metformin $(0,62.5,125 \mu \mathrm{mol} / \mathrm{L})$ or alendronate $(0,62.5,125 \mathrm{nmol} / \mathrm{L})$, modulate osteoclast differentiation for 5 days. Then, total RNA extraction and subsequent experiments were performed as described above. The primer sequences are listed in Table 1.

\section{Table 1 Primer sequences used in qRT-PCR}




\begin{tabular}{|c|c|c|}
\hline Gene & Froward $\left(5^{\prime}-3^{\prime}\right)$ & Reverse $\left(5^{\prime}-3^{\prime}\right)$ \\
\hline Sox9 & GAGCCGGATCTGAAGAGGGA & GCTTGACGTGTGGCTTGTTC \\
\hline Aggrecan & CAGTGGGATGCAGGCTGGCT & CCTCCGGCACTCGTTGGCTG \\
\hline ADAMTS5 & GGAGCGAGGCCATTTACAAC & CGTAGACAAGGTAGCCCACTTT \\
\hline MMP3 & ACATGGAGACTTTGTCCCTTTTG & TTGGCTGAGTGGTAGAGTCCC \\
\hline MMP13 & СTTCTTCTTGTTGAGCTGGACTC & CTGTGGAGGTCACTGTAGACT \\
\hline CTSK & CCAGTGGGAGCTATGGAAGA & AAGTGGTTCATGGCCAGTTC \\
\hline DC-STAMP & TTCATCCAGCATTTGGGAGT & ACAGAAGAGAGCAGGGCAAC \\
\hline AcP5 & CAGCAGCCAAGGAGGACTAC & ACATAGCCCACACCGTTCTC \\
\hline NFATC1 & CAACGCCCTGACCACCGATAG & GGCTGCCTTCCGTCTCATAGT \\
\hline c-Fos & TTTCAACGCCGACTACGAGG & GCGCAAAAGTCCTGTGTGTT \\
\hline TNFRSF11 & GAAGATGCTTTGGTGGGTGT & TCAGTCGGGATCAGTGTGAG \\
\hline $18 S$ & CGGCTACCACATCCAAGGAA & GCTGGAATTACCGCGGCT \\
\hline
\end{tabular}

\section{Western blotting}

To evaluate the expression of AMPK signaling pathway factors during the anti-inflammatory effect of metformin, we inoculated ATDC5 cells in a 6-well plate. Then, we added IL-1 $\beta$ to the wells after pretreating the cells with metformin $(0,5 \mathrm{mmol} / \mathrm{L})$ for $2 \mathrm{~h}$. Then, total protein was extracted from ATDC5 cells at 30 min using radioimmunoprecipitation assay (RIPA) lysis buffer (consisting of phosphatase inhibitors, and $100 \mathrm{mmol} / \mathrm{P}$ PMSF). To examine the expression of osteoarthritis-related proteins, ATDC5 cells were cultured in 6-well plates with various concentrations of metformin $(0,2,4$, and $5 \mathrm{mmol} / \mathrm{L})$ with or without IL-1 $1 \beta(10 \mathrm{ng} / \mathrm{mL})$ for 5 days.

Similar to the real-time PCR assay, total protein was extracted from osteoclasts with radioimmunoprecipitation assay (RIPA) lysis buffer (consisting of phosphatase inhibitors, and $100 \mathrm{mmol} / \mathrm{PMSF}$ ). In addition, the signaling pathway-related proteins associated with osteoclast differentiation were analyzed at $0 \mathrm{~min}, 10 \mathrm{~min}, 20 \mathrm{~min}, 30 \mathrm{~min}, 60 \mathrm{~min}$, and $120 \mathrm{~min}$ after 50 $\mathrm{ng} / \mathrm{mL}$ RANKL was added to each well, which had been pretreated with metformin $(0,125 \mu \mathrm{M})$ for $2 \mathrm{~h}$. The protein concentration was quantified using a BCA protein assay kit (BOSTER, Wuhan, China). Then, $10 \%$ sodium dodecyl sulfate-polyacrylamide gel electrophoresis was used to resolve the proteins. The separated proteins were transferred to polyvinylidene difluoride (PVDF) membranes and subsequently blocked with $5 \%$ nonfat milk for $2 \mathrm{~h}$. The samples were then incubated overnight at $4{ }^{\circ} \mathrm{C}$ with the following primary antibodies: anti-SOX9 antibody (1:5000, Cat. \#ab185966), anti-aggrecan (1:200, 
Cat. \#absc166951), anti-MMP3 (1:500, Cat. \#AF0217), anti-MMP13 (1:500, Cat. \#AF5355), anti-AMPK alpha 1 (1:200, Cat. \#10929-2-AP), anti-phospho-AMPK alpha 1 (1:500, Cat. \#AF3422), anti-cathepsin K (1:200, Cat. \#sc48353), anti-NFATc1 (1:200, Cat. \#sc7294), anti-c-Fos (1:500, Cat. \#AF0132), anti-NF-kB (anti-p65) (1:500, Cat. \#BF0382), anti-phospho-NF-kB (anti-pp65) (1:500, Cat. \#AF2006). The membranes were washed three times with Tris-buffered saline containing 0.1\% Tween-20 (TBST) 15 min each time) and incubated with HRP-conjugated secondary antibodies at room temperature for 1 hour. After being washed with TBST three times, the membranes underwent ECL development. We visualized the immunoreactive bands by measuring the gray values with ImageJ.

\section{Histological and Immunohistochemical Analysis}

The surgical side or normal joints from the mice were fixed in $4 \%$ paraformaldehyde and embedded in paraffin. Serial 6- $\mu \mathrm{m}$-thick sections were cut and collected on glass slides. Then, the samples were stained with hematoxylin and eosin (H\&E) or safranin O/fast green (SO/FG). The degeneration of articular cartilage was scored by the Osteoarthritis Research Society International (OARSI) guidelines[19]. TRAP-positive cells attached to subchondral bone were stained with TRAP. For immunohistochemistry, 6- $\mu \mathrm{m}$ sagittal sections were prepared from the paraffin-embedded tissue and treated with the following primary antibody anti-MMP13 (1:500, Cat. \#AF5355), anti-phospho-AMPK alpha 1 (1:500, Cat. \#AF3422), anti-Col II (1:100, Cat. \#ab34712) overnight at $4^{\circ} \mathrm{C}$. Then, the cells were incubated with anti-rabbit secondary antibodies for $1 \mathrm{~h}$ at $37^{\circ} \mathrm{C}$ and examined by $\mathrm{DAB}$.

\section{Immunofluorescence staining}

ATDC5 cells were maintained in 6-well plates containing coverslips and stimulated with5 $\mathrm{mmol} / \mathrm{L}$ metformin with or without $10 \mathrm{ng} / \mathrm{ml} \mathrm{IL}-1 \beta$ for $48 \mathrm{~h}$. Then, the coverslips were fixed in $4 \%$ formaldehyde for 30 min and permeabilized with $0.1 \%$ Triton X-100 for $20 \mathrm{~min}$. The coverslips were blocked with goat serum at $37^{\circ} \mathrm{C}$ for 1 hour. Next, the coverslips were incubated with the following primary antibodies: anti-SOX9 antibody (1:100, Cat. \#ab185966), and anti-MMP13 (1:500, Cat. \#AF5355) overnight at $4{ }^{\circ} \mathrm{C}$. After three washes with PBS, the cells were incubated with fluorescent secondary antibodies. DAPI was used for nuclear staining. Fluorescence inverted microscopy (CKX53; Olympus, Tokyo, Japan) was used to obtain immunofluorescent images.

\section{Detection of reactive oxygen species (ROS) in vitro}

For intracellular ROS analysis, Reactive oxygen species assay kit (Solarbio, Beijing, China) was used. ATDC5 cells were pretreated with $5 \mathrm{mmol} / \mathrm{L}$ metformin, incubated with $10 \mu \mathrm{mol} / \mathrm{L}$ 2',7'-dichlorofluorescein diacetate (DCF-DA) for 20 minutes in the dark, and then washed three times with DMEM. Next, the cells were stimulated with IL-1 $\beta$ for 5 min. ROS production was observed by fluorescence inverted microscopy. M-CSF-induced BMMs were seeded at a density of 1.5×105/well in 6-well cell culture plates and stimulated with $50 \mathrm{ng} / \mathrm{ml}$ RANKL without or with $125 \mu \mathrm{M}$ metformin for 3 days. Then, the cells were incubated with $10 \mu \mathrm{mol} / \mathrm{L}$ DCF-DA for 20 minutes in the dark, and ROS production was examined by Fluorescence inverted microscopy as described above. 


\section{Statistical analysis}

All quantitative data from multiple experiments were calculated by GraphPad Prism 8 and are presented as the mean \pm standard deviation (SD). The experiments were repeated three times. One-way analysis of variance was used to calculate the $p$ value, and a $p$ value of $<0.05$ was considered to indicate a statistically significant result.

\section{Results}

\section{Metformin inhibited IL-1 $\beta$-induced chondrocyte matrix catabolism in ATDC5 cells}

It has been shown that IL-1 $\beta$ can inhibit the metabolism of the chondrocyte matrix. In high-density culture experiments, treatment by IL-1 $\beta$ resulted in a significant reduction in Alcian blue staining as assessed by the staining area multiplied by the grey value, whereas the metformin co-stimulation group had abundant Alcian blue staining, indicating that matrix degradation was significantly inhibited by $5 \mathrm{mmol} / \mathrm{L}$ metformin. On the other hand, there was no difference between the control and metformin-treated groups, regardless of the concentration of metformin (Fig. 1a, b). Immunofluorescence results also showed that 5 $\mathrm{mmol} / \mathrm{L}$ metformin inhibited the IL-1 $\beta$-induced increased expression of MMP13 and decreased expression of SOX9 in ATDC5 cells(Fig. 1d). The CCK-8 assay demonstrated that 2, 4, and $5 \mathrm{mmol} / \mathrm{L}$ metformin had no significant effect on ATDC5 cell viability. When ATDC5 cells were treated with both IL$1 \beta$ and 2,4 , and $5 \mathrm{mmol} / \mathrm{L}$ metformin, the proliferation of ATDC5 cells was promoted at $48 \mathrm{~h}$ (Fig. 1c).

Real-time PCR showed that the expression of matrix metalloproteinase 3 (MMP3) and matrix metalloproteinase 13 (MMP3) was upregulated by IL-1 $\beta$. However, when cells were stimulated with both IL-1 $\beta$ and metformin, the result was altered (Fig. 2a,2b). Consistent with the real-time PCR results, western blot analysis showed that the expression of MMP13 and MMP3 was increased in ATDC5 cells treated with IL-1 $\beta$ (Fig. 2e), while SRY-box transcription Factor 9 (SOX9) and aggrecan expression was decreased (Fig. 2e). Metformin decreased IL-1 $\beta$-induced MMP3 and MMP13 generation but enhanced sox 9 and aggrecan expression, although Real-time PCR showed no statistical difference in sox9 and aggrecan expression (Fig. 2c, 2d). We suggest that this may be because protein degradation is inhibited by metformin. These data suggest that metformin plays an anti-inflammatory role in IL-1 $\beta$-induced ATDC5 cells.

\section{Metformin protected against IL-1ß-driven catabolism in cartilage explants}

To explore whether metformin could modulate extracellular matrix (ECM) generation during OA progression, "relatively normal" human cartilage explants were cultured in the presence or absence of metformin with or without IL-1 $\beta$ for $24 \mathrm{~h}, 48 \mathrm{~h}$, and $72 \mathrm{~h}$. After being cultured, proteoglycan content, which was determined by SO/FG staining, significantly decreased in the IL-1 $\beta$ treatment group compared with the control group, especially at $72 \mathrm{~h}$. Moreover, the proteoglycan content was restored by $5 \mathrm{mmol} / \mathrm{L}$ metformin. Immunohistological staining showed that metformin enhanced the type II collagen (COL II), and reduced the MMP13 contents after 3 days of treatment (Fig.3). 


\section{Metformin and alendronate inhibited RANKL-induced osteoclast differentiation}

Before examining the effects of metformin and alendronate on osteoclast differentiation, we tested the cytotoxicity of these drugs on BMMs using a CCK-8 assay. The results demonstrated that metformin did not inhibit cell proliferation at concentrations of 0-250 $\mu \mathrm{mol} / \mathrm{L}$ (Fig. 4a). Then, BMMs were incubated with metformin and stimulated with M-CSF plus RANKL. TRAP staining was used to evaluate reactive osteoclast formation. TRAP-positive cells containing more than three nuclei were considered osteoclasts (Fig. 4b, 4c). Our results indicated that BMMs incubated with metformin $(62.5 \mu \mathrm{mol} / \mathrm{L}, 125 \mu \mathrm{mol} / \mathrm{L})$ had fewer TRAP-positive cells than BMMs incubated without metformin. This result revealed that metformin had an inhibitory effect on osteoclast differentiation. We also found that osteoclast formation was lower in groups treated with $125 \mathrm{nmol} / \mathrm{L}$ and $62.5 \mathrm{nmol} / \mathrm{L}$ alendronate than in the control group, indicating that alendronate inhibited osteoclast differentiation (Fig. 4b, 4c). Moreover, the CCK-8 assay showed no significant difference in the viability of BMMs after treatment with different concentrations of alendronate, suggesting that alendronate has no toxic effect on living cells at concentrations of 0-125 nmol/L. (Fig. 4a).

\section{Metformin and alendronate downregulated the expression of osteoclast differentiation-related genes}

Because cellular oncogene FOS (c-Fos), dendritic cell-specific transmembrane protein (DC-STAMP), TNFRSF11, and cathepsin K (CTSK) are critical genes associated with RANKL-induced osteoclast differentiation, we examined the effects of metformin and alendronate on osteoclast differentiation at the genetic level by real-time PCR. The results showed that osteoclast function-related genes, such as CTSK, were upregulated by RANKL treatment (Fig. 3a), and DC-STAMP, which is associated with osteoclast fusion, was similarly upregulated (Fig. 3a). Metformin and alendronate together decreased the RANKL-induced mRNA expression of target genes (Fig. 3a). TNFRSF11 expression was enhanced by RANKL, and this gene encodes the RANKL protein. Our results showed that the expression of the TNFRSF11 gene was downregulated in osteoclasts in the metformin-treated and alendronatetreated groups (Fig. 3a). These data confirm that metformin and alendronate both have inhibitory effects on RANKL-induced osteoclast formation and osteoclast function.

\section{NFATc1 expression was inhibited by metformin and alendronate}

The nuclear factor of activated T cells cl (NFATc1) induction is a hallmark event in determining the fate of osteoclasts, and NFATc1 activation induces the transcription of osteoclast-specific genes such as CTSK[20]. We measured the expression of NFATc1, and CTSK by western blotting. We found that metformin inhibited the expression of these two proteins compared to that in cells treated with RANKL alone (Fig. 3b, 3c). we investigated the expression of c-Fos, the key transcription factors in osteoclastogenesis. Metformin inhibited the RANKL-induced c-Fos generation (Fig. 3a, 3b, 3c). This suppression was also induced by alendronate (Fig.3a, 3b, 3c).

Metformin inhibited inflammation and osteoclast differentiation via the AMPK pathway, NF-KB pathway 
As metformin is a known AMPK activator[21], we examined whether metformin upregulated the expression and phosphorylation of AMPKa1 in IL-1 $\beta$-treated ATDC5 cells using western blotting. IL-1 $\beta$ significantly reduced the levels of phosphorylated AMPKa1, while metformin $(5 \mathrm{mmol} / \mathrm{L})$ reversed the effect on AMPKa1 phosphorylation at $2 \mathrm{~h}$ (Fig. 6a). Moreover, the addition of metformin reversed the inflammatory response caused by IL-1 $\beta$ in the ATDC 5 cell line. These results were confirmed in the mouse knee joint by immunohistochemistry. The expression of phosphorylated AMPKa1 in the articular chondrocytes of knee cartilage was decreased after DMM surgery, and metformin enhanced AMPKa1 phosphorylation in articular chondrocytes after DMM surgery (Fig. 8a, 8d). During RANKL-induced osteoclastogenesis, the decrease in p-AMPK levels was reversed by 125 $\mu \mathrm{mol} / \mathrm{L}$ metformin at $10 \mathrm{~min}, 20 \mathrm{~min}, 30 \mathrm{~min}, 60 \mathrm{~min}$, and $120 \mathrm{~min}$. Moreover, nuclear factor-KB (NF-KB) activation, which is a key molecular event in early RANKL-induced osteoclastogenesis, was significantly inhibited by metformin in the presence of RANKL at $20 \mathrm{~min}, 30 \mathrm{~min}, 60 \mathrm{~min}$, and $120 \mathrm{~min}$ (Fig. 6f).

\section{Metformin inhibited oxidative stress in ATDC5 cells and BMMs}

Because oxidative stress has been linked to the pathogenesis of osteoarthritis due to its role in chondrocyte apoptosis and extracellular matrix (ECM) degradation[22], we determined whether metformin could suppress IL-1 $\beta$-induced oxidative stress in ATDC 5 cells by measuring ROS production. We found that pretreatment with metformin $(5 \mathrm{mmol} / \mathrm{L})$ significantly inhibited IL-1 $\beta$-mediated production of ROS in OA chondrocytes (Fig.6b). Osteoclast formation and activity are induced by elevated ROS. In our study, ROS levels in BMMs treated with metformin plus RANKL were significantly lower than those in RANKLtreated BMMs and were comparable to basal levels in untreated BMMs (Fig.6k). Therefore, these data indicate that metformin may possess antioxidant properties.

\section{Combined treatment with metformin and alendronate limited OA development in the DMM mouse model through chondroprotective effects and inhibiting subchondral bone remodeling}

The DMM mouse model was used to validate the protective effects of alendronate plus metformin on OA development. The overall structure of the knee joint and the loss of cartilage were examined by H\&E, SO, and immunohistochemistry staining. As expected, slightly stripped cartilage and less safranin 0 staining were observed in the DMM group (Fig.7a). However, the amount of cartilage damage in the alendronatetreated group was lower than that in the DMM group. Moreover, less cartilage damage was observed in the combined alendronate and metformin treatment group than in the other groups (Fig. 7a). The OARSI score in the combined alendronate and metformin treatment group was significantly lower than that in the DMM group (Fig. 7b). Similarly, we used immunohistochemistry to show that the expression of MMP13 was dramatically increased in the DMM group, and the significantly increased expression of MMP13 in cartilage was attenuated by combined treatment with alendronate and metformin. But, the expression of COL II was enhanced (Fig.8a, 8b, 8c). We also measured the number of osteoclasts in the subchondral bone by TRAP staining. After surgery, the number of osteoclasts was increased in the subchondral bone in the DMM group. This effect was abrogated by alendronate, and this preventive effect was further enhanced by combined treatment with alendronate and metformin (Fig. 7a, 7c). 


\section{Discussion}

Alendronate, a bisphosphonate, is a bone turnover inhibitor that is used to treat osteoarthritis due to its ability to protect chondrocytes by inhibiting matrix metalloproteinase expression and subchondral bone reconstruction [23]. A trial to evaluate bisphosphonates as structural modifiers in osteoarthritis nears completion. The future use of this therapy is trending to less frequent administration because of the potential for excessive inhibition of conversion with prolonged use, and interactions with anabolic therapies are currently being examined. In the present study, we found that alendronate combined with metformin delayed the progression of OA in mice by inhibiting inflammation and attenuating RANKLinduced bone resorption. Mechanistically, metformin suppressed osteoclastogenesis and improved cartilage matrix synthesis by reducing ROS production and mediating AMPK signaling, and NF-KB signaling.

IL-1 $\beta$ was used to establish an invitro inflammation model in ATDC5 chondrocytes and induced cartilage matrix degradation, which was shown by Alcian blue staining. Features of osteoarthritis include degradation of the extracellular matrix (ECM) and loss of cartilage gene expression in articular cartilage. As expected, in response to IL-1 $\beta$, the expression of aggrecan and SOX9 was decreased, while matrix metalloproteinases (MMP3 and MMP13), which are enzymes that can degrade all components of the ECM, were upregulated in the ATDC5 chondrocytes. MMP13 is a substrate-specific enzyme whose overexpression can lead to cartilage degeneration, as it degrades the proteoglycans aggrecan and collagen, both of which are components of articular cartilage[24]. The expression of MMP3 is increased in arthritis, and this protein mainly degrades non-collagenous matrix components of the joint. In our study, we found that the upregulation of MMP3 and MMP13 expression by IL-1 $\beta$ was eliminated by metformin. SOX9, a master regulator of Aggrecan and collagen, plays roles in both cartilage development and homeostasis by directly targeting genes encoding cartilage-specific extracellular matrix components[25]. Therefore, modifying the level of Sox9 expression in OA cartilage may be beneficial in improving the course of OA. Our results showed that metformin upregulated SOX9 expression, which was inhibited by IL-1 $\beta$. Aggrecan is one of the main structures in the extracellular matrix of articular cartilage, and it is well known that the loss of Aggrecan accelerates the progression of osteoarthritis[26]. As we hypothesized, metformin abrogated the inhibitory effect of IL-1 $\beta$ on Aggrecan. Consistent with the results of cellular experiments, treatment with inflammatory cytokines (IL-1 $\beta$ ) caused cartilage tissue degradation. Metformin treatment reduced the degree of S/F staining loss in human articular cartilage explants cultured in the presence of IL-1 $\beta$. Furthermore, metabolism in the cartilage explants remained stable for up to $72 \mathrm{~h}$, and there was no significant difference in the proteoglycan content in the absence of IL-1 $\beta$ at 24,48 , and $72 \mathrm{~h}$. These results suggest that metformin may play a protective role in $\mathrm{OA}$.

Currently, numerous studies suggest that changes in the microarchitecture of subchondral bone precede articular cartilage degradation in OA[13]. Early-stage increased bone loss in subchondral bone is an important component of the pathogenetic process that leads to OA[13]. In our experimental model, DMM$\mathrm{OA}$ mice treated with alendronate plus metformin exhibited reduced numbers of TRAP ${ }^{+}$multinucleated osteoclasts and articular cartilage degeneration with improved OARSI scores compared to DMM-OA mice. 
This is the result of the inhibition of osteoclast formation and bone resorption by alendronate in combination with metformin. There was a downward trend between the alendronate-treated and untreated groups but no significant difference. This outcome may be the result of the low-frequency effect of low concentrations of alendronate. We also found that both metformin and alendronate inhibited RANKL-induced osteoclast formation in vitro. In this study, RANKL-induced osteoclasts were confirmed by TRAP staining, and the expression of osteoclast marker genes and proteins, including c-Fos, NFATCl, CTSK, and DC-STAMP, was quantitatively examined by western blotting and RT-PCR. CTSK, which is specifically expressed by osteoclasts[27], is essential for osteoclast-mediated bone resorption[28]. NFATcl, which is the transcription factor that is most potently induced by RANKL, works together with other transcription factors to induce various osteoclast-specific genes[29]. The TRAP, calcitonin receptor, and cathepsin $\mathrm{K}$ genes, all of which are important for osteoclast function, are regulated by NFATC1. DC-STAMP, which was shown to be essential for the cell-cell fusion of osteoclast precursor cells, has been shown to be a transcriptional target of NFATc1. The c-Fos, which is an indispensable downstream regulatory factor of RANKL, accelerates the formation of osteoclasts by activating the downstream factor NFATc1 [30]. In this study, both metformin and alendronate inhibited the expression of these factors. Thus, metformin and alendronate may inhibit osteoclast formation by downregulating the expression of these genes.

We further investigated the mechanism by which metformin inhibits inflammation and osteoclasts in osteoarthritis. AMP-activated protein kinase (AMPK) is a key molecule in the antidiabetic mechanism of metformin and is also involved in the signaling pathways associated with bone physiology. AMPK has three subunits $(a, \beta, \gamma)$, and its catalytic activity is associated with the a subunit[31]. Phosphorylation of Ser486 in the a subunit triggers AMPK activation. In OA human articular cartilage, decreased AMPKa phosphorylation was observed compared to that in the normal cartilage group[32]. In our study, the protein expression of $\mathrm{p}-\mathrm{AMPKa} 1$ was significantly inhibited by IL-1 $\beta$. This inhibition, as well as MMP expression, was reversed after treatment with metformin, and these results suggest that the antiinflammatory effects of metformin involve the AMPK signaling pathway. Conversely, studies have shown a negative regulatory effect of AMPK on cartilage differentiation[8]. In line with this, our experiments showed that the expression of SOX9, a marker gene of cartilage differentiation, was repressed in the presence of metformin. Aggrecan expression was also suppressed. This finding suggests that AMPK activity may have a protective effect on the degradation of articular cartilage. Moreover, impairments in AMPK contribute to mitochondrial dysfunction. ROS, which is increased in response to mitochondrial dysfunction, promotes cartilage degradation in OA by breaking down collagen, aggregating proteoglycans, and activating matrix metalloproteinases (MMPs)[33] and is also connected to AMPK signaling. Our in vitro studies showed an increase in ROS in IL-1 $\beta$-treated inflammatory chondrocytes. In the presence of metformin, phosphorylated AMPKa1 abrogated the increased ROS level. Our data reveal that metformin-induced reductions in ROS production and AMPK phosphorylation may be protective mechanisms in chondrocytes. AMPK is a negative regulator of RANKL. The binding of RANKL to its receptor RANK leads to the activation of the c-Fos signaling pathway, which activates NFATc1, a transcription factor associated with osteoclast differentiation, fusion, and activation[34]. Evidence has 
shown that RANKL induces the phosphorylation/activation of AMPK-a in BMMs and stimulates the formation of TRAP-positive multinucleated cells[35]. There are two different $a$ isoforms, $a 1$, and a2, and the a1-isomer is mainly expressed in BMMs. We hypothesized that metformin could inhibit RANKLinduced osteoclast formation in the presence of M-CSF by activating/phosphorylating AMPKa1. Consistently, in our study, NFATc1 and c-Fos were significantly reduced by metformin after treatment with RANKL, while metformin-induced AMPKa1 phosphorylation was maintained. Thus, metformin plays an important role as a negative regulator of RANKL-mediated osteoclastogenesis by acting as an inhibitor of NFATc1 activation through c-Fos and AMPK signaling. It has also been shown that the increase in ROS in BMMs can be attenuated by metformin[36]. ROS can be involved in osteoclast formation through various signaling pathways, including the MAPK, PI3K, and NF-KB pathways[37]. In our study, metformin pretreatment reduced the RANKL-induced increase in ROS levels in BMMs. This finding suggests that the regulation of intracellular ROS levels may be a target by which metformin inhibits osteoclast differentiation. Regardless, osteoarthritis is considered a whole-joint disease that involves both chondrocytes and osteoclasts, and metformin-mediated targeting ROS and inhibiting inflammation through AMPK activation will greatly slow the progression of osteoarthritis. We further showed that metformin regulated the NF-KB signaling pathway. Persistently elevated responses in the NF-KB pathway are associated with diseases such as rheumatoid arthritis (RA) and $\mathrm{OA}[38]$. Activation of the transcription factor NF-KB in turn activates genes that regulate osteoclast differentiation[39]. In our study, metformin inhibited RANKL-induced NF-KB phosphorylation in osteoclasts. Although it remains unclear how metformin-mediated AMPK signaling affects NF-KB, we suspect that metformin exerts a therapeutic effect on OA through the AMPK and NF-KB pathways.

Alendronate similarly inhibited osteoclast differentiation and bone resorption. We found that alendronate downregulated the expression of c-Fos and NFATc1, suggesting that alendronate may inhibit the damaging effects of OCs on subchondral bone by interfering with the c-Fos-NFATc1 pathway.

\section{Conclusion}

In conclusion, metformin combined with alendronate inhibited inflammatory factors and osteoclast differentiation, improved cartilage matrix metabolism and subchondral bone reconstruction, and delayed the progression of $O A$ in mice. Metformin combined with alendronate may be a useful treatment for attenuating early-stage $\mathrm{OA}$. This would be an ideal drug/drug combination for treating osteoarthritis in the presence of osteoporosis combined with T2D. In addition, this approach needs to be further validated in a clinical setting.

\section{Declarations}

\section{Acknowledgments}

We thanked the Key Laboratory of Bone and Soft Tissue Injury Repair, The Second Hospital of Shanxi Medical University for platform support. 


\section{Funding}

This project was supported by the National Natural Science Foundation of China (Grant No. 81572207, 81871815), Natural Science Fund of Shanxi Province (Grant No. 20210302123284), Health Commission of Shanxi Province (Grant Nos. 2020XM54, 2020075), and the Shanxi Province emerging industry-leading talent project.

\section{Competing interests}

The authors declare that they have no competing interests.

\section{Authors' contributions}

Yanjing Guo and Shaowei Wang contributed to conception. Yanjing Guo, Chunfang Wang, and Shaowei Wang designed the study, and Yanjing Guo wrote the first draft of the manuscript. Sujing Zong, Zhenyu Wang, and Jiyu Zhao organized the database. Ruijia Yang, Chunfang Wang, and Chongwei Chen performed the statistical analysis. Ruijia Yang, and Chongwei Chen wrote sections of the manuscript. All authors read and approved the final manuscript.

\section{Ethics approval and consent to participate}

The study was reviewed and approved by the Ethics Committee of Shanxi Medical University (2018LL039).

\section{Availability of data and materials}

The datasets used and/or analyzed during the current study are available from the corresponding author upon reasonable request.

\section{References}

1. Hu W, Chen Y, Dou C, Dong S: Microenvironment in subchondral bone: predominant regulator for the treatment of osteoarthritis. Ann Rheum Dis 2020.

2. Glyn-Jones S, Palmer AJ, Agricola R, Price AJ, Vincent TL, Weinans H, Carr AJ: Osteoarthritis. Lancet 2015, 386(9991):376-387.

3. Louati K, Vidal C, Berenbaum F, Sellam J: Association between diabetes mellitus and osteoarthritis: systematic literature review and meta-analysis. RMD Open 2015, 1(1):e000077.

4. Paschou SA, Dede AD, Anagnostis PG, Vryonidou A, Morganstein D, Goulis DG: Type 2 Diabetes and Osteoporosis: A Guide to Optimal Management. J Clin Endocrinol Metab 2017, 102(10):3621-3634.

5. Vestergaard P: Risk of newly diagnosed type 2 diabetes is reduced in users of alendronate. Calcif Tissue Int 2011, 89(4):265-270. 
6. Lu CH, Chung CH, Lee CH, Hsieh CH, Hung YJ, Lin FH, Tsao CH, Hsieh PS, Chien WC: Combination COX-2 inhibitor and metformin attenuate rate of joint replacement in osteoarthritis with diabetes: $A$ nationwide, retrospective, matched-cohort study in Taiwan. PLoS One 2018, 13(1):e0191242.

7. Chen Y, Qiu F, Yu B, Chen Y, Zuo F, Zhu X, Nandakumar KS, Xiao C: Metformin, an AMPK Activator, Inhibits Activation of FLSs but Promotes HAPLN1 Secretion. Mol Ther Methods Clin Dev 2020, 17:1202-1214.

8. Bandow K, Kusuyama J, Kakimoto K, Ohnishi T, Matsuguchi T: AMP-activated protein kinase (AMPK) activity negatively regulates chondrogenic differentiation. Bone 2015, 74:125-133.

9. Terkeltaub R, Yang B, Lotz M, Liu-Bryan R: Chondrocyte AMP-activated protein kinase activity suppresses matrix degradation responses to proinflammatory cytokines interleukin-1beta and tumor necrosis factor alpha. Arthritis Rheum 2011, 63(7):1928-1937.

10. Zhou S, Lu W, Chen L, Ge Q, Chen D, Xu Z, Shi D, Dai J, Li J, Ju H et al: AMPK deficiency in chondrocytes accelerated the progression of instability-induced and ageing-associated osteoarthritis in adult mice. Sci Rep 2017, 7:43245.

11. Li J, Zhang B, Liu WX, Lu K, Pan H, Wang T, Oh CD, Yi D, Huang J, Zhao L et al: Metformin limits osteoarthritis development and progression through activation of AMPK signalling. Ann Rheum Dis 2020, 79(5):635-645.

12. Tang Y, Hong C, Cai Y, Zhu J, Hu X, Tian Y, Song X, Song Z, Jiang R, Kang F: HIF-1alpha Mediates Osteoclast-Induced Mandibular Condyle Growth via AMPK Signaling. J Dent Res 2020, 99(12):13771386.

13. Burr DB, Gallant MA: Bone remodelling in osteoarthritis. Nat Rev Rheumato/2012, 8(11):665-673.

14. Zhu S, Zhu J, Zhen G, Hu Y, An S, Li Y, Zheng Q, Chen Z, Yang Y, Wan M et al: Subchondral bone osteoclasts induce sensory innervation and osteoarthritis pain. J Clin Invest 2019, 129(3):1076-1093.

15. Fang H, Huang L, Welch I, Norley C, Holdsworth DW, Beier F, Cai D: Early Changes of Articular Cartilage and Subchondral Bone in The DMM Mouse Model of Osteoarthritis. Sci Rep 2018, 8(1):2855.

16. Kadri A, Ea HK, Bazille C, Hannouche D, Liote F, Cohen-Solal ME: Osteoprotegerin inhibits cartilage degradation through an effect on trabecular bone in murine experimental osteoarthritis. Arthritis Rheum 2008, 58(8):2379-2386.

17. Zhang L, Hu H, Tian F, Song H, Zhang Y: Enhancement of subchondral bone quality by alendronate administration for the reduction of cartilage degeneration in the early phase of experimental osteoarthritis. Clin Exp Med 2011, 11(4):235-243.

18. Lenart BA, Lorich DG, Lane JM: Atypical fractures of the femoral diaphysis in postmenopausal women taking alendronate. N Engl J Med 2008, 358(12):1304-1306.

19. Glasson SS, Chambers MG, Van Den Berg WB, Little CB: The OARSI histopathology initiative recommendations for histological assessments of osteoarthritis in the mouse. Osteoarthritis Cartilage 2010, 18 Suppl 3:S17-23. 
20. An J, Hao D, Zhang Q, Chen B, Zhang R, Wang Y, Yang H: Natural products for treatment of bone erosive diseases: The effects and mechanisms on inhibiting osteoclastogenesis and bone resorption. Int Immunopharmacol 2016, 36:118-131.

21. Saisho Y: Metformin and Inflammation: Its Potential Beyond Glucose-lowering Effect. Endocr Metab Immune Disord Drug Targets 2015, 15(3):196-205.

22. Lepetsos P, Papavassiliou KA, Papavassiliou AG: Redox and NF-kappaB signaling in osteoarthritis. Free Radic Biol Med 2019, 132:90-100.

23. Hayami T, Pickarski M, Wesolowski GA, McLane J, Bone A, Destefano J, Rodan GA, Duong LT: The role of subchondral bone remodeling in osteoarthritis: reduction of cartilage degeneration and prevention of osteophyte formation by alendronate in the rat anterior cruciate ligament transection model. Arthritis Rheum 2004, 50(4):1193-1206.

24. Mehana EE, Khafaga AF, El-Blehi SS: The role of matrix metalloproteinases in osteoarthritis pathogenesis: An updated review. Life Sci 2019, 234:116786.

25. Lefebvre V, Dvir-Ginzberg M: SOX9 and the many facets of its regulation in the chondrocyte lineage. Connect Tissue Res 2017, 58(1):2-14.

26. Ouyang Y, Wang W, Tu B, Zhu Y, Fan C, Li Y: Overexpression of SOX9 alleviates the progression of human osteoarthritis in vitro and in vivo. Drug Des Devel Ther 2019, 13:2833-2842.

27. Wen X, Yi LZ, Liu F, Wei JH, Xue Y: The role of cathepsin $\mathrm{K}$ in oral and maxillofacial disorders. Oral Dis 2016, 22(2):109-115.

28. Dodds RA, James IE, Rieman D, Ahern R, Hwang SM, Connor JR, Thompson SD, Veber DF, Drake FH, Holmes $\mathrm{S}$ et al: Human osteoclast cathepsin $\mathrm{K}$ is processed intracellularly prior to attachment and bone resorption. J Bone Miner Res 2001, 16(3):478-486.

29. Negishi-Koga T, Takayanagi H: Ca2+-NFATc1 signaling is an essential axis of osteoclast differentiation. Immunol Rev 2009, 231(1):241-256.

30. Qu Y, Liu X, Zong S, Sun H, Liu S, Zhao Y: Protocatechualdehyde Inhibits the Osteoclast Differentiation of RAW264.7 and BMM Cells by Regulating NF-kappaB and MAPK Activity. Biomed Res Int 2021, 2021:6108999.

31. Bahrambeigi S, Yousefi B, Rahimi M, Shafiei-Irannejad V: Metformin; an old antidiabetic drug with new potentials in bone disorders. Biomed Pharmacother 2019, 109:1593-1601.

32. Petursson F, Husa M, June R, Lotz M, Terkeltaub R, Liu-Bryan R: Linked decreases in liver kinase B1 and AMP-activated protein kinase activity modulate matrix catabolic responses to biomechanical injury in chondrocytes. Arthritis Res Ther 2013, 15(4):R77.

33. Zhao X, Petursson F, Viollet B, Lotz M, Terkeltaub R, Liu-Bryan R: Peroxisome proliferator-activated receptor gamma coactivator 1alpha and Fox03A mediate chondroprotection by AMP-activated protein kinase. Arthritis Rheumatol 2014, 66(11):3073-3082.

34. Kim JY, Min JY, Baek JM, Ahn SJ, Jun HY, Yoon KH, Choi MK, Lee MS, Oh J: CTRP3 acts as a negative regulator of osteoclastogenesis through AMPK-c-Fos-NFATc1 signaling in vitro and RANKLinduced calvarial bone destruction in vivo. Bone 2015, 79:242-251. 
35. Lee YS, Kim YS, Lee SY, Kim GH, Kim BJ, Lee SH, Lee KU, Kim GS, Kim SW, Koh JM: AMP kinase acts as a negative regulator of RANKL in the differentiation of osteoclasts. Bone 2010, 47(5):926-937.

36. Xian H, Liu Y, Rundberg Nilsson A, Gatchalian R, Crother TR, Tourtellotte WG, Zhang Y, AlemanMuench GR, Lewis G, Chen W et al: Metformin inhibition of mitochondrial ATP and DNA synthesis abrogates NLRP3 inflammasome activation and pulmonary inflammation. Immunity 2021, 54(7):1463-1477 e1411.

37. Tao H, Ge G, Liang X, Zhang W, Sun H, Li M, Geng D: ROS signaling cascades: dual regulations for osteoclast and osteoblast. Acta Biochim Biophys Sin (Shanghai) 2020, 52(10):1055-1062.

38. Baker RG, Hayden MS, Ghosh S: NF-kappaB, inflammation, and metabolic disease. Cell Metab 2011, 13(1):11-22.

39. Park JH, Lee NK, Lee SY: Current Understanding of RANK Signaling in Osteoclast Differentiation and Maturation. Mol Cells 2017, 40(10):706-713.

\section{Figures}

\section{Figure 1}

Metformin enhanced anabolism and inhibited IL-1 $\beta$-induced chondrocyte matrix catabolism in ATDC5 cells and is not cytotoxic in vitro. (a) Alcian blue staining was quantified. (b) Alcian blue staining of ATDC5 cells. (c) Effects of metformin on ATDC5 cells viability by the CCK-8 assay. $N=3 .{ }^{\star} P<0.05,{ }^{\star * \star} P<$ $0.001, * \star \star \star P<0.0001$ vs. control group (without IL-1 $\beta$ induction and metformin treatment). (d) Immunofluorescence analyses were used to determine the protein level of sox9 and MMP13 in ATDC5 cells. Scale bars, $20 \mu \mathrm{m}$. $\mathrm{N}=3$. Data are presented as mean \pm standard deviation of the mean $(S D)$. ${ }^{\star P}<$ $0.05,{ }^{*} \mathrm{P}<0.01,{ }^{*} * \star * \mathrm{P}<0.0001$ vs. IL-1 $\beta$ group. Statistical analysis was conducted using a one-way analysis of variance.

\section{Figure 2}

Metformin alleviates IL-1 $\beta$-induced degradation of the extracellular matrix. (a - d) The expression level of (a) MMP13, (b) MMP3, (c) Aggrecan, and (d) SOX9 mRNA in ATDC5 cells were determined by qRT-PCR. (e) The expression level of Aggrecan, Sox9, MMP3, and MMP13 protein in ATDC5 cells were determined by western blot. ( $f-i)$ Quantitative analysis of the differential expression level of ( $f$ ) Aggrecan, (g) SOX9, (h) MMP3, and (i) MMP13. The GAPDH was selected as the control protein. $\mathrm{N}=3$. Data are presented as mean \pm standard deviation of the mean (SD). ${ }^{*} P<0.05$, ${ }^{*} \mathrm{P}<0.01$, ${ }^{*} * \star \mathrm{P}<0.0001$ vs. IL-1 $\beta$ group. Statistical analysis was conducted using one-way analysis of variance. 


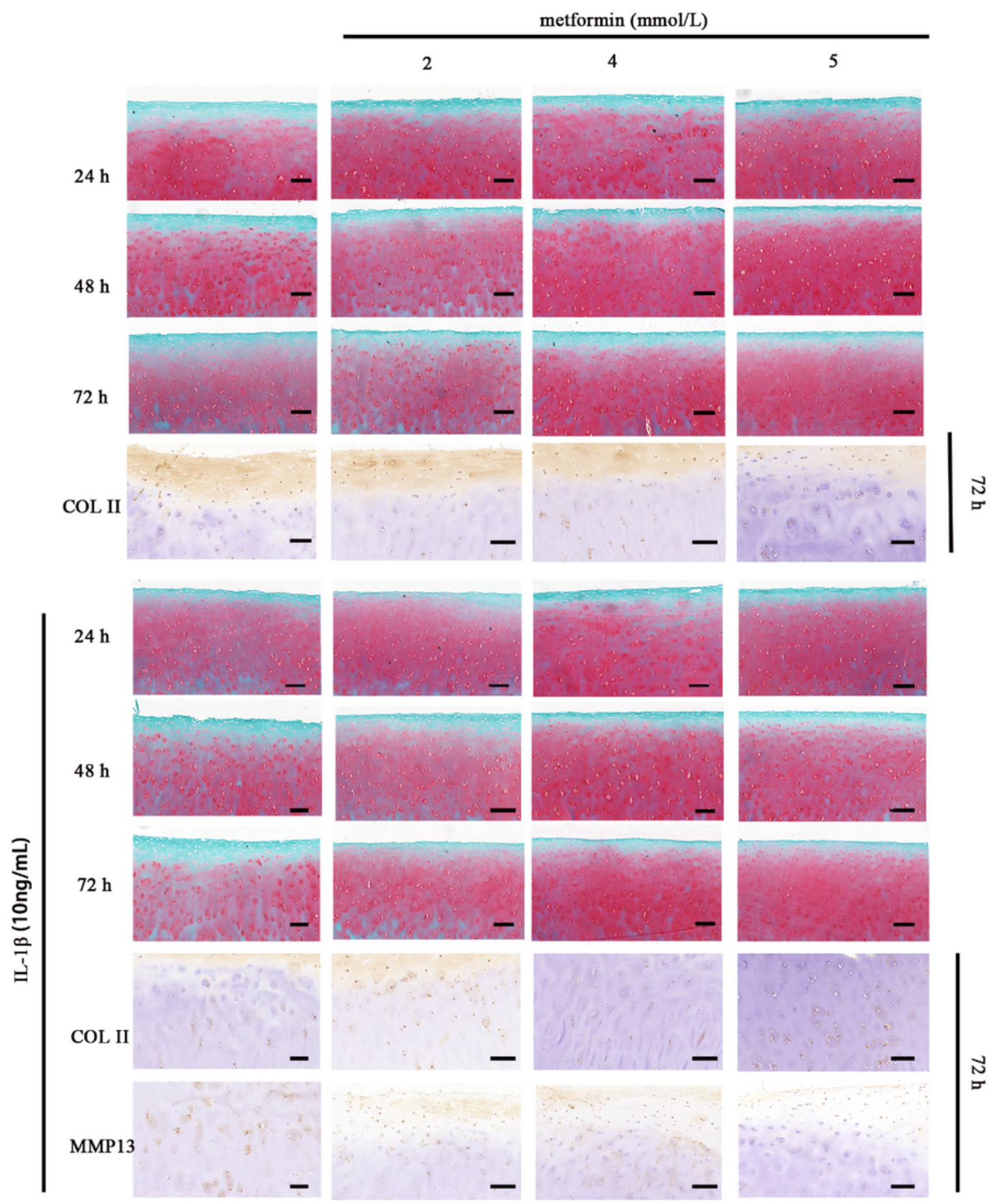

Figure 3

Metformin protected against IL-1 $\beta$-driven ECM degradation in cartilage explants. The proteoglycan content of cartilage explants was measured by safranin 0-fast green staining after $24 \mathrm{~h}, 48 \mathrm{~h}$, and $72 \mathrm{~h}$ of incubation with metformin or metformin + IL-1 $\beta$. Immunohistochemistry staining of COLII and MMP13 in control, IL-1 $\beta$, metformin, metformin + IL-1 $\beta$ group after $72 \mathrm{~h}$ (safranin O-fast green stain, scale bars, 100 $\mu \mathrm{m}$. COLII, MMP13, scale bars, $50 \mu \mathrm{m})$. $\mathrm{N}=3$. 


\section{Figure 4}

Metformin and alendronate inhibit in vitro osteoclastogenesis. (a) Effects of metformin and alendronate on BMMs viability by the CCK-8 assay. (b) Quantification analysis of TRAP-positive multinucleated cells (MNCs, nuclei $\geq 3$ ). (c) TRAP staining shows the numbers of TRAP-positive cells ( $>3$ nuclei) in BMMs treated with $50 \mathrm{ng} / \mathrm{mL} \mathrm{M}-\mathrm{CSF}, 50 \mathrm{ng} / \mathrm{mL}$ RANKL, and metformin or alendronate. $\mathrm{N}=3$. Data are presented as mean \pm standard deviation of the mean (SD), ${ }^{\star \star \star \star} \mathrm{P}<0.0001 \mathrm{vs}$. RANKL group. Statistical analysis was conducted using one-way analysis of variance.

\section{Figure 5}

Metformin and alendronate inhibit in vitro osteoclastogenesis and suppresses osteoclastogenesis-related gene and protein expression. (a) qRT-PCR analysis of the mRNA levels of osteoclastogenesis-related genes, c-Fos, CTSK, DC-STAMP, and TNFRSF11. The 18sRNA was selected as the control gene. (b) Quantification of the ratios of band intensity of NFATc1, c-Fos, and CTSK relative to GAPDH. (c) Western blotting was used to determine the protein level of NFATc1, c-Fos, and CTSK. N=3. Data are presented as mean \pm standard deviation of the mean (SD), ${ }^{*} P<0.05,{ }^{* * P}<0.01$, ${ }^{* *} \mathrm{P}<0.001$, ${ }^{* \star * *} \mathrm{P}<0.0001$ vs. RANKL group. Statistical analysis was conducted using one-way analysis of variance.

\section{Figure 6}

Metformin reduced oxidative stress as well as inhibited inflammation and osteoclast differentiation via the AMPK signaling pathway, and NF-KB signaling pathway. (a) Western blot assay was performed to detect the protein expression level of AMPKa1, and p-AMPKa1 in ATDC5 cells. (b)Representative confocal images of IL-1 $\beta$-induced ROS production in ATDC5 cells in the presence or absence of pre-treated metformin. (c - d) Quantification of the ratios of band intensity of AMPKa1, and p-AMPKa1 relative to GAPDH. (e) The ratios of band intensity of p-AMPKa1 / The ratios of band intensity of AMPKa1. $N=3$, *P $<0.05$ vs. IL-1 $\beta$ group. (f) Western blot images show the levels of AMPKa1, p-AMPKa1, NF-KB-p65, and pNF-kB-p65 at 0, 10, 20, 30, 60, and 120 mins in the RANKL- and RANKL + metformin-treated BMMs. (g - j) Quantification of the ratios of band intensity of (g) AMPKa1, (h) p-AMPKa1, (i) NF-KB-p65, and (j) p-NFKB-p65 relative to GAPDH. (k) Representative confocal images of RANKL-induced ROS production in BMMs with and without pre-treated of metformin. $\mathrm{N}=3$. Data are presented as mean \pm standard deviation of the mean (SD), ${ }^{\star} P<0.05,{ }^{\star *} P<0.01,{ }^{\star} * \star * P<0.0001$ vs. RANKL group. Statistical analysis was conducted using one-way analysis of variance. 
a

DMM
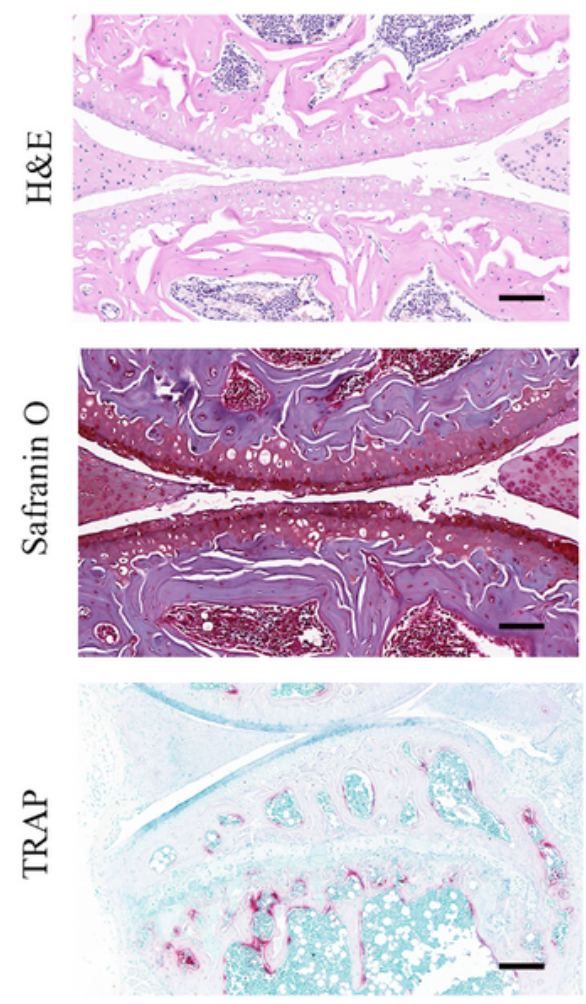

b

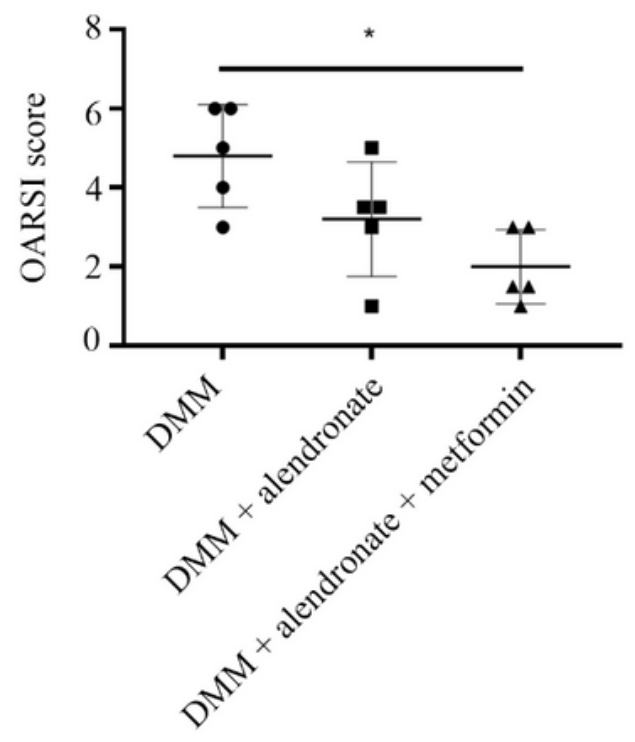

$\mathrm{DMM}+$ alendronate
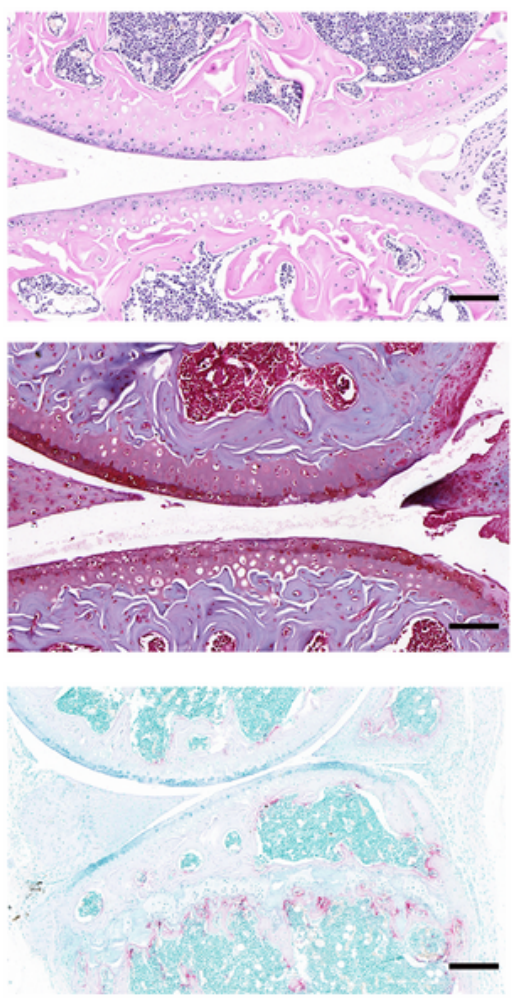

$\mathrm{DMM}+$ alendronate + metformin
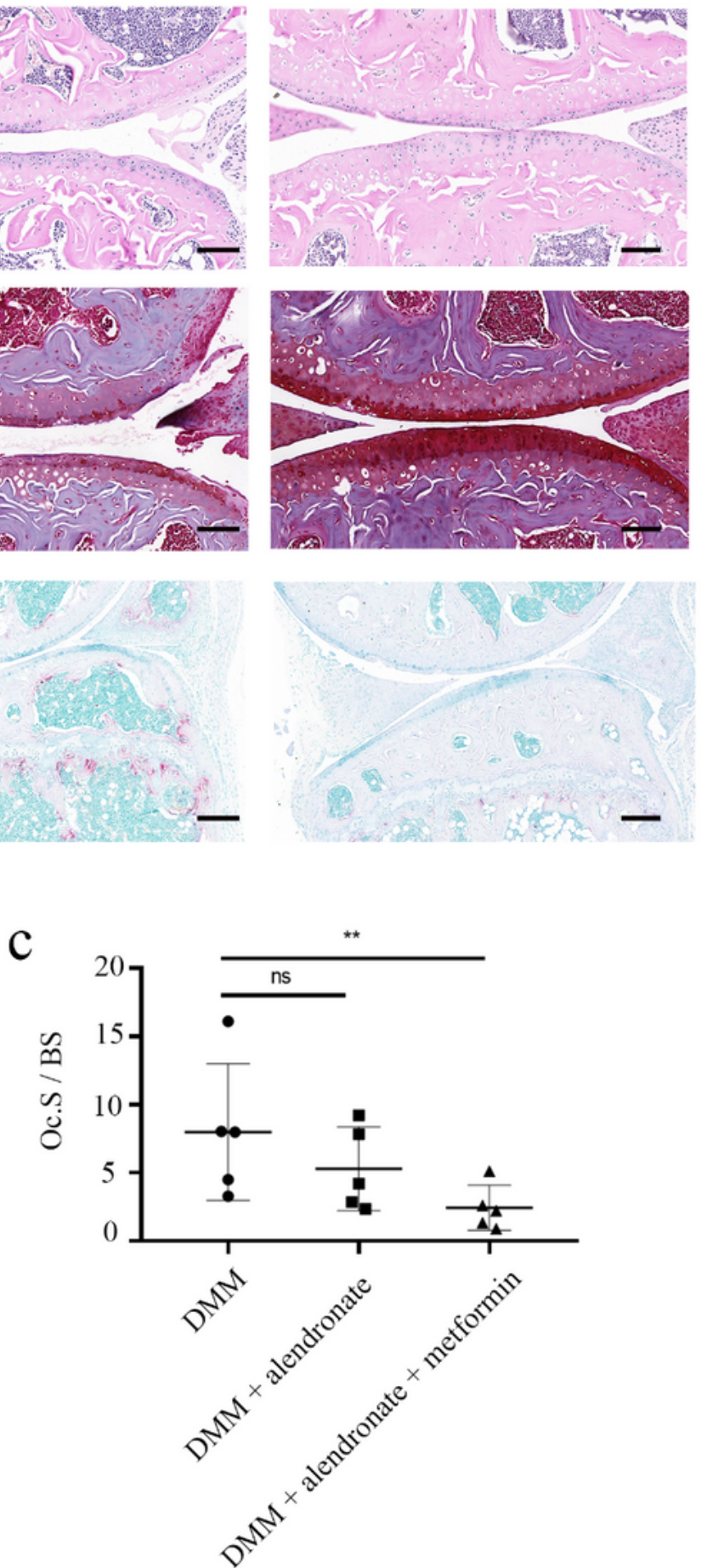

\section{Figure 7}

Combined treatment with metformin and alendronate limited OA development in the DMM mouse model through chondroprotective effects and inhibiting subchondral bone remodeling. (a) Representative images of H\&E, safranin O, TRAP staining in DMM, alendronate-treated, alendronate + metformin-treated mice after $8 \mathrm{w}$ of administration. (b) The corresponding Osteoarthritis Research Society International (OARSI) scores from DMM, alendronate-treated, and alendronate + metformin-treated mice. (c) 
Quantitative measurements of Oc.S / BS in the no-treatment group, alendronate-treated group, and alendronate + metformin-treated group. $(d-f)$ The number of COLII, MMP13, and p-AMPKa1 positive cells in the knee cartilage. (j) Immunohistochemistry staining of COLII, MMP13, p-AMPKa1 in DMM, alendronate-treated, alendronate + metformin-treated mice after $8 \mathrm{w}$ of administration. TRAP staining, scale bar, $100 \mu \mathrm{m} ; \mathrm{H} \& \mathrm{E}$, safranin $\mathrm{O}$ staining, scale bar, $50 \mu \mathrm{m}$. $\mathrm{N}=5$. Data are presented as mean \pm standard deviation of the mean (SD), ${ }^{\star} P<0.05,{ }^{\star} \mathrm{P} P<0.01,{ }^{\star} * \star * \mathrm{P}<0.0001$ vs. DMM group. Statistical analysis was conducted using one-way analysis of variance.

\section{Figure 8}

Combined treatment with metformin and alendronate limited OA development and induced phosphorylation and expression of AMP-activated protein kinase (AMPK) in the DMM mouse model. Immunohistochemistry staining of COLII, MMP13, p-AMPKa1 in DMM, alendronate-treated, alendronate + metformin-treated mice after $8 \mathrm{w}$ of administration. (a) Immunohistochemistry staining, scale bar, $50 \mu \mathrm{m}$ (upper panel) and $20 \mu \mathrm{m}$ (lower panel). (b-d) The number of (b) COLII, (c) MMP13, and (d) p-AMPKa1 positive cells in the knee cartilage. $\mathrm{N}=5$. Data are presented as mean \pm standard deviation of the mean $(\mathrm{SD}),{ }^{*} \mathrm{P}<0.05,{ }^{\star} * \mathrm{P}<0.01,{ }^{\star \star \star *} \mathrm{P}<0.0001$ vs. DMM group. Statistical analysis was conducted using oneway analysis of variance.

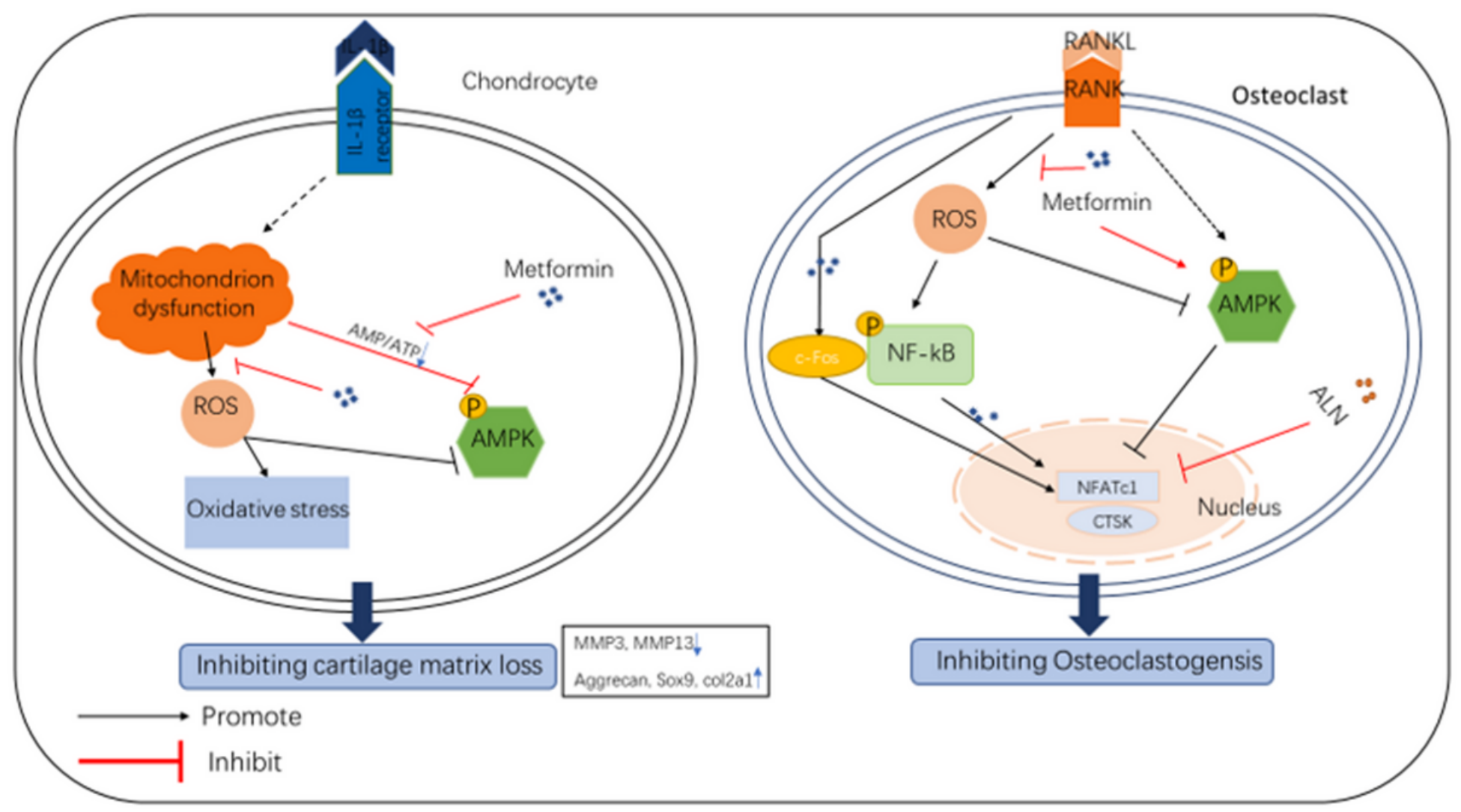

Figure 9 
Mechanisms by which metformin and alendronate limit the progression of osteoarthritis by targeting chondrocytes and osteoclasts. 